\title{
Evaluation of patients with diffuse unilateral subacute neuroretinitis by spectral domain optical coherence tomography with enhanced depth imaging
}

This article was published in the following Dove Press journal:

Clinical Ophthalmology

5 June 2014

Number of times this article has been viewed

\author{
Rodrigo F Berbel \\ Antonio Marcelo B Casella' \\ Eduardo C de Souza ${ }^{2}$ \\ Michel E Farah² \\ 'State University of Londrina, \\ Londrina, ${ }^{2}$ Federal University \\ of São Paulo, São Paulo, Federative \\ Republic of Brazil
}

Background: The purpose of this study was to determine the value of spectral domain optical coherence tomography (SD-OCT) in assessing patients with diffuse unilateral subacute neuroretinitis (DUSN).

Methods: This was an observational case series with clinical imaging correlation performed at the Retina and Vitreous Institute of Londrina and State University of Londrina, Paraná, Brazil. The series comprised ten consecutive patients with a confirmed diagnosis of DUSN, ie, seven patients with late-stage disease and three with early-stage disease, who were assessed by SD-OCT for mean macular, retinal nerve fiber layer, and choroidal thickness using enhanced depth imaging software.

Results: Comparing the affected eye with the healthy fellow eye, significant diffuse atrophy of the retinal layers with a decrease in mean macular $(P=0.004)$ and retinal nerve fiber layer $(P=0.002)$ thickness was found in all cases. There was no difference in choroidal thickness $(P=0.262)$.

Conclusion: The correlation of SD-OCT results with central vision and funduscopic findings may explain the profound loss of visual function in patients with DUSN.

Keywords: eye infections, parasitic, retinitis, optical coherence tomography, diffuse unilateral subacute neuroretinitis, enhanced depth imaging

\section{Introduction}

Diffuse unilateral subacute neuroretinitis (DUSN) is caused by a retinal nematode. ${ }^{1}$ DUSN appears in the early stages as multifocal choroiditis and optic nerve inflammation, and can develop into the chronic form, with optic nerve atrophy, retinal vessel narrowing, and diffuse changes in the retinal pigmented epithelium. ${ }^{2}$ It is very important to make the diagnosis in the early phase when laser photocoagulation of the worm or oral albendazole has the greatest chance of success. ${ }^{2,3}$

DUSN is still often difficult to diagnose in the acute phase. The differential diagnosis is based on exclusion of a number of causes of multifocal choroiditis, such as multiple evanescent white dot syndrome, ${ }^{4}$ sarcoidosis, vascular occlusive disease, and unilateral retinitis pigmentosa, as well as other infectious causes, including syphilitic chorioretinitis and toxoplasmosis. ${ }^{5}$

In addition to this difficulty, complementary examinations only indicate nonspecific changes. Some are more invasive, such as fluorescein angiography, which shows diffuse changes in the retinal pigmented epithelium and also dye leaks at the optic nerve. Indocyanine green angiography shows hypofluorescent spots corresponding to areas of inflammation. These procedures may produce adverse reactions and are often difficult to perform in children. ${ }^{1,6}$

\author{
Correspondence:Antonio Marcelo \\ B Casella \\ State University of Londrina, \\ Av Bandeirantes, 500, 86010-010, \\ Londrina, Federative Republic of Brazil \\ Tel +55 433324 II 77 \\ Fax +554333240790 \\ Email mcasella@sercomtel.com.br
}

submit your manuscript | www.dovepress.com

Dovepress

http://dx.doi.org//0.2147/OPTH.S60302 
Spectral-domain optical coherence tomography (SD-OCT) is a noninvasive test that is reproducible, repeatable, and easy to carry out. It also provides useful information when studying the vitreoretinal interface and various retinal structures, including the retinal nerve fiber layer, optic nerve, and now the choroid by a new method known as enhanced depth imaging. ${ }^{\text {? }}$

The aim of this study was to determine the value of SD-OCT in evaluation of DUSN and to understand the pathogenesis of the disease. We focused specifically on description of the damage, localization of the worm, and follow-up features.

\section{Materials and methods}

A cross-sectional observational study was conducted at the State University of Londrina and Retina and Vitreous Institute of Londrina, Brazil, between October 2011 and January 2011. Ten confirmed cases of DUSN were evaluated. There were three acute cases, one with a history of 3 weeks of unilateral visual loss and two cases previously treated in the acute phase, and seven chronic cases with visual loss for a few months to years, all of whom were treated at diagnosis. The study was approved by the institutional ethics committee of the State University of Londrina and followed the tenets of the Declaration of Helsinki. Permission to review medical charts was obtained from the institutional review board/ethics committee of the State University of Londrina.

The patients were evaluated by full ophthalmologic examination, including color fundus photography, near infrared wavelength fundus autofluorescence, and SD-OCT (Heidelberg Spectralis ${ }^{\circledR}$, Heidelberg Engineering, Germany) in affected and healthy eyes.
We determined the OCT parameters of mean macular thickness and choroidal thickness using the enhanced depth imaging program (version 5.2), with $200 \times 200$ axial scans, an area of $6 \times 6 \mathrm{~mm}$, and of retinal nerve fiber layer thickness with a circular scan pattern of 12 degrees centered on the optic nerve. The choroidal thickness measurement was manually segmented (by RFB) using Heidelberg Eye Explore software with the caliper function (Heidelberg Spectralis). The choroidal area between the outer border of the retinal pigmented epithelium and the inner sclera border was considered, and five values were assigned. The retinal nerve fiber layer was revised and also corrected manually (by RFB) when the OCT software was not reliable in recognizing those margins, usually due to substantial atrophy in the affected eye. To facilitate comparison, only the central macular and global average retinal nerve fiber layer thickness and subfoveal choroidal thickness values were analyzed. Only images with scores higher than 25 were considered.

Statistical analysis was performed using Statistical Package for the Social Sciences version 19 software (SPSS Inc, Chicago, IL, USA), whereby tomographic measurements were evaluated by the Wilcoxon test for dependent samples, comparing the affected eye with the healthy eye. $P$-value $<0.05$ was considered to be statistically significant.

\section{Results}

The ten patients in this study comprised six males and four females with a mean age of $18 \pm 4.37$ years. One patient presented with an acute picture, having 3 weeks of visual loss, pallid optic disc, venous narrowing, and visual acuity of counting fingers. The worm was found with some difficulty

Table I Clinical data according to age, sex, stage, visual acuity before and after treatment: choroidal, macular and retinal nerve fiber layer thickness

\begin{tabular}{|c|c|c|c|c|c|c|c|c|c|c|}
\hline \multirow[t]{2}{*}{ Case } & \multirow[t]{2}{*}{ Sex } & \multirow[t]{2}{*}{ Stage } & \multirow[t]{2}{*}{ Initial VA } & \multirow[t]{2}{*}{ Final VA } & \multicolumn{2}{|c|}{$\mathrm{CT}(\mu \mathrm{m})$} & \multicolumn{2}{|c|}{ MT $(\mu \mathrm{m})$} & \multicolumn{2}{|c|}{ RNFL $(\mu \mathrm{m})$} \\
\hline & & & & & $\overline{\mathrm{AE}}$ & HE & $\overline{\mathrm{AE}}$ & HE & $\overline{\mathrm{AE}}$ & HE \\
\hline I & $\mathrm{F}$ & Acute & $\mathrm{CF}$ & $20 / 40$ & 333 & 331 & 210 & 264 & 63 & 84 \\
\hline 2 & $\mathrm{~F}$ & Chronic & $\mathrm{CF}$ & $\mathrm{CF}$ & 351 & 325 & 206 & 285 & 64 & 86 \\
\hline 3 & $\mathrm{~F}$ & Chronic & $\mathrm{CF}$ & $\mathrm{CF}$ & 276 & 332 & 152 & 253 & 54 & 98 \\
\hline 4 & $M$ & Chronic & $\mathrm{CF}$ & $20 / 400$ & $34 I$ & 395 & 212 & 281 & 75 & 99 \\
\hline 5 & $M$ & Chronic & $20 / 400$ & $20 / 200$ & 267 & 267 & 236 & 279 & 85 & 100 \\
\hline 6 & $M$ & Chronic & $\mathrm{CF}$ & $\mathrm{CF}$ & 279 & 305 & 205 & 310 & 51 & 109 \\
\hline 7 & M & Chronic & $\mathrm{CF}$ & $\mathrm{CF}$ & 311 & 386 & 218 & 293 & 78 & 109 \\
\hline 8 & $M$ & Chronic & $\mathrm{CF}$ & $20 / 400$ & 372 & 333 & 128 & 263 & 23 & 129 \\
\hline 9 & $M$ & Acute & $\mathrm{CF}$ & $20 / 50$ & 217 & 181 & 258 & 311 & 45 & 86 \\
\hline \multirow[t]{4}{*}{10} & $\mathrm{~F}$ & Acute & $\mathrm{CF}$ & $20 / 20$ & 268 & 366 & 271 & 264 & 59 & 82 \\
\hline & & & & Mean & 302 & 322 & 210 & 280 & 59.7 & 98.2 \\
\hline & & & & $S D$ & 47.9 & 62.2 & 43.3 & 20 & 18 & 14.7 \\
\hline & & & & $P$ & 0.262 & & 0.004 & & 0.002 & \\
\hline
\end{tabular}

Abbreviations: CF, counting fingers; VA, visual acuity; CT, choroidal thickness; MT, macular thickness; RNFL, retinal nerve fiber layer; AE, affected eye; HE, healthy eye; $M$, male; F, female SD, standard deviation; $P, P$-value. 


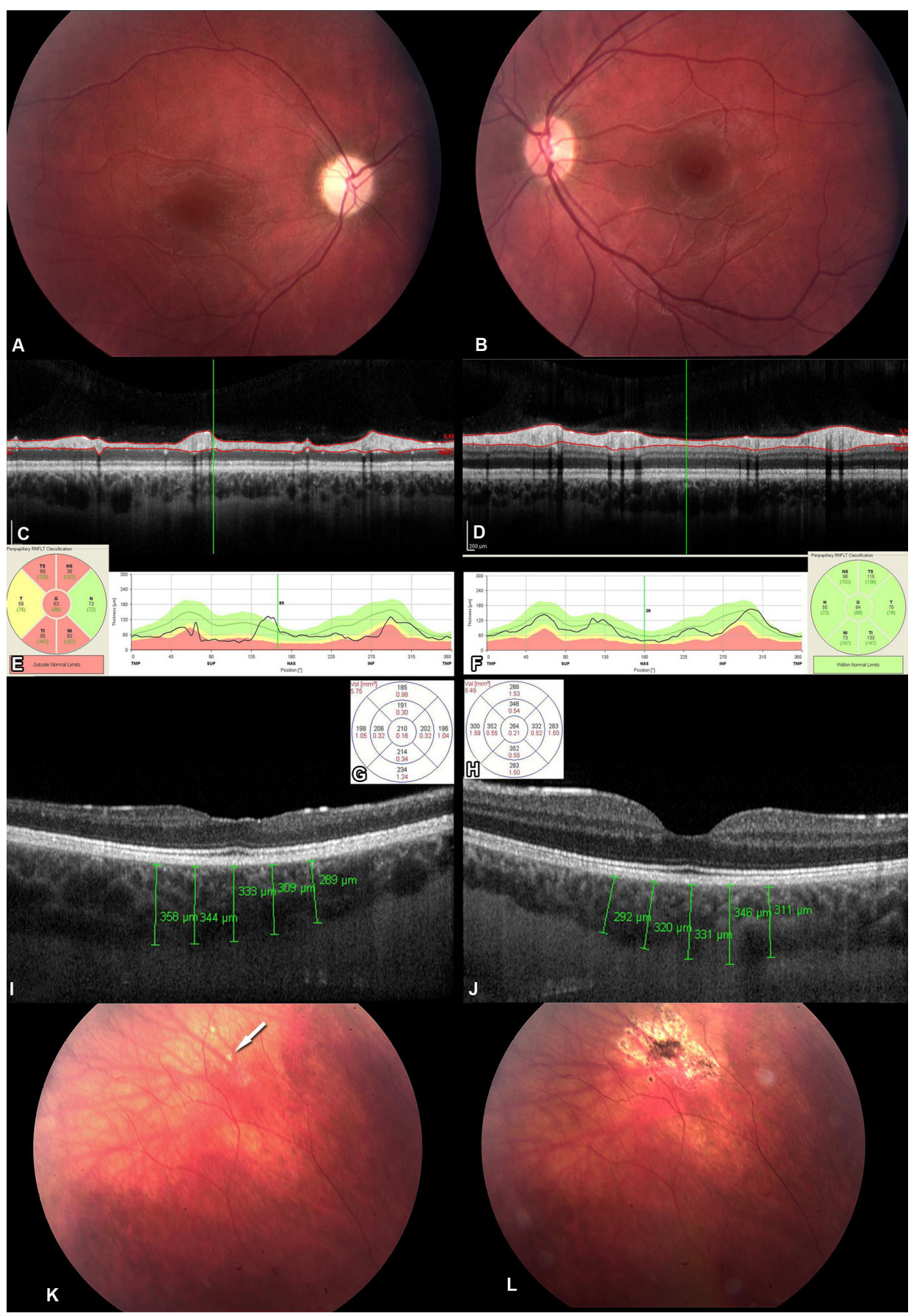

Figure I Affected eye (A) and healthy eye (B) for case I. Note the decrease in thickness of the retinal nerve fiber layer in the affected eye (C, E) in comparison with the contralateral eye (D, F). Decrease in mean macular thickness in the affected eye versus the healthy eye $(\mathbf{G}, \mathbf{H})$, and an image of choroidal thickness using enhanced depth imaging software, where little difference is noted between the affected eye and the healthy eye (I, J). Fundus images show the peripheral localization of the worm (see white arrow) (K) and its destruction after treatment with laser photocoagulation (L).

Abbreviations: TMP, temporal; SUP, superior; NAS, nasal; INF, inferior; RNFLT, retinal nerve fiber layer thickness; TS, temporal-superior; T, temporal; TI, temporalinferior; G, global average; NS, nasal-superior; N, nasal; NI, nasal-inferior.

in the temporal periphery of the retina and photocoagulated. After treatment, there was recovery of visual acuity to 20/40, but a clear loss of retinal nerve fiber layer thickness persisted (Figure 1).

Two other cases presented with a visual acuity of counting fingers and a history of visual loss over some months. The fundus photograph was compatible with DUSN, and a motile nematode was found in both patients and photocoagulated. In one of these cases, an intraretinal worm shape was detected on OCT, appearing as a hyperreflective object of irregular shape affecting all layers of the retina (Figure 2).

In seven further cases, the worm had already been photocoagulated, and the patients were evaluated in relation to the chronic effects of DUSN (Figure 3). In two of these 


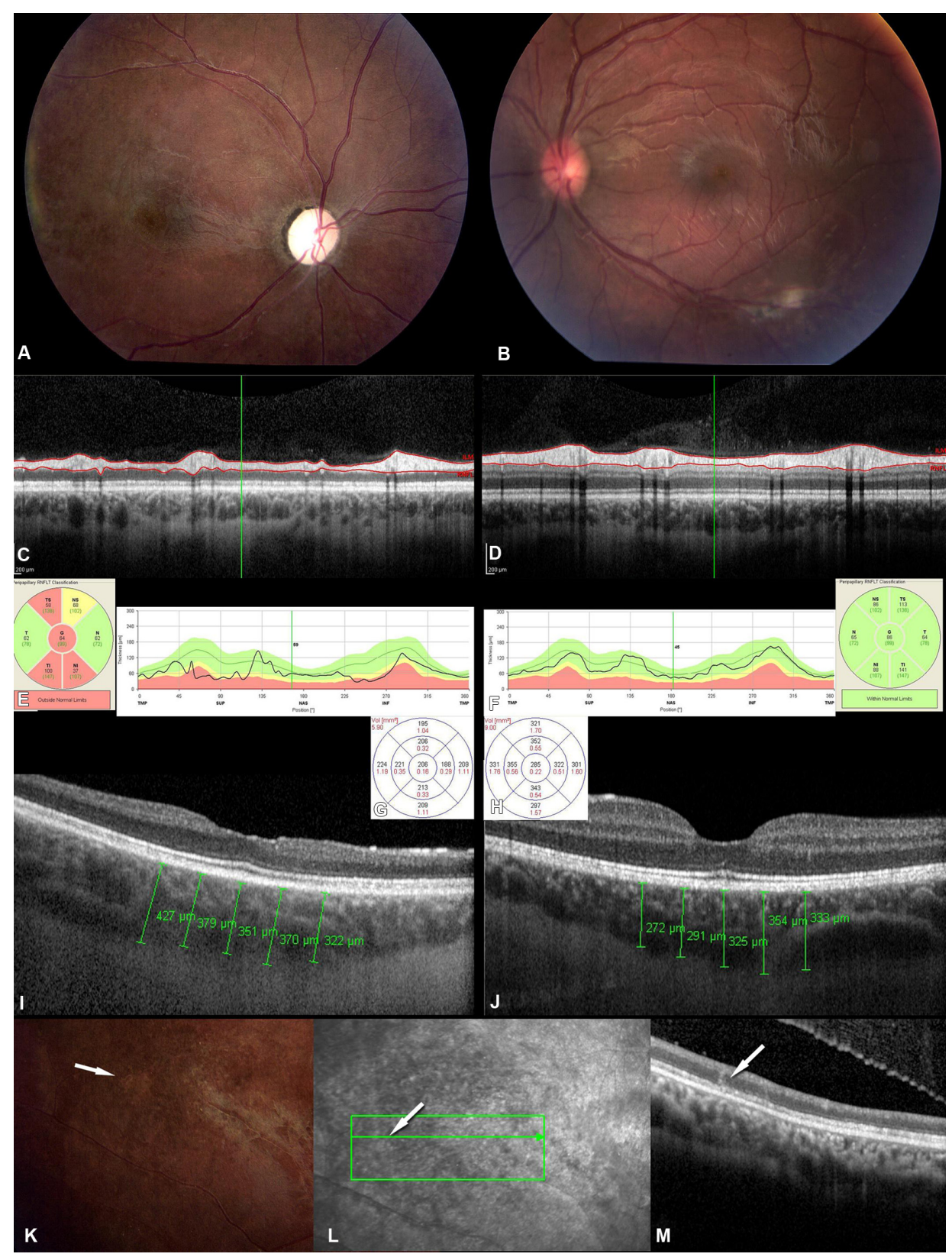

Figure 2 Case 2 had visual loss over some months, with counting fingers at the initial assessment. Note the atrophy of the retinal nerve fiber layer with a decrease in overall thickness in the affected eye (A, C, E) in comparison with the healthy eye (B, D, F). Substantial atrophy is indicated by the decrease in mean macular thickness (G, $\mathbf{H})$ but without significant changes in the thickness of the choroid (I, J). Fundus photography $(\mathbf{K})$ indicated localization of a motile worm (see white arrow) that was treated by laser photocoagulation. Evaluation by optical coherence tomography (L, $\mathbf{M})$ shows intraretinal localization (white arrow) in the outer retina but affecting all layers of the retina. Abbreviations: TMP, temporal; SUP, superior; NAS, nasal; INF, inferior; RNFLT, retinal nerve fiber layer thickness; TS, temporal-superior; T, temporal; TI, temporalinferior; G, global average; NS, nasal-superior; N, nasal; NI, nasal-inferior.

cases, the worm was treated with photocoagulation at the onset of symptoms, with the patients achieving good final visual acuity (cases 9 and 10).

Determination of mean macular thickness demonstrated a decrease in thickness in the whole retina in the affected eye compared with the fellow eye. Mean macular thickness in affected eyes was $209.6 \pm 43.32 \mu \mathrm{m}$ compared with $280.3 \pm 19.66 \mu \mathrm{m}$ in healthy eyes, and the difference was statistically significant $(P=0.004)$. Evaluation of retinal nerve fiber layer thickness demonstrated a notable and significant decrease in the affected eyes (mean 59.7 $\pm 17.98 \mu \mathrm{m}$ versus $98.2 \pm 14.71 \mu \mathrm{m}$ for healthy eyes; $P=0.002$ ). Choroidal thickness did not vary significantly between the two eyes, although thickness was less in the majority of affected eyes (mean 301.5 $\pm 47.92 \mu \mathrm{m}$ versus $322.1 \pm 62.24 \mu \mathrm{m}$ for healthy eyes; $P=0.262$ ). (See Table 1 ).

\section{Discussion}

DUSN is a disease that results in irreversible visual loss, so it is very important to make the diagnosis in the early stages. 


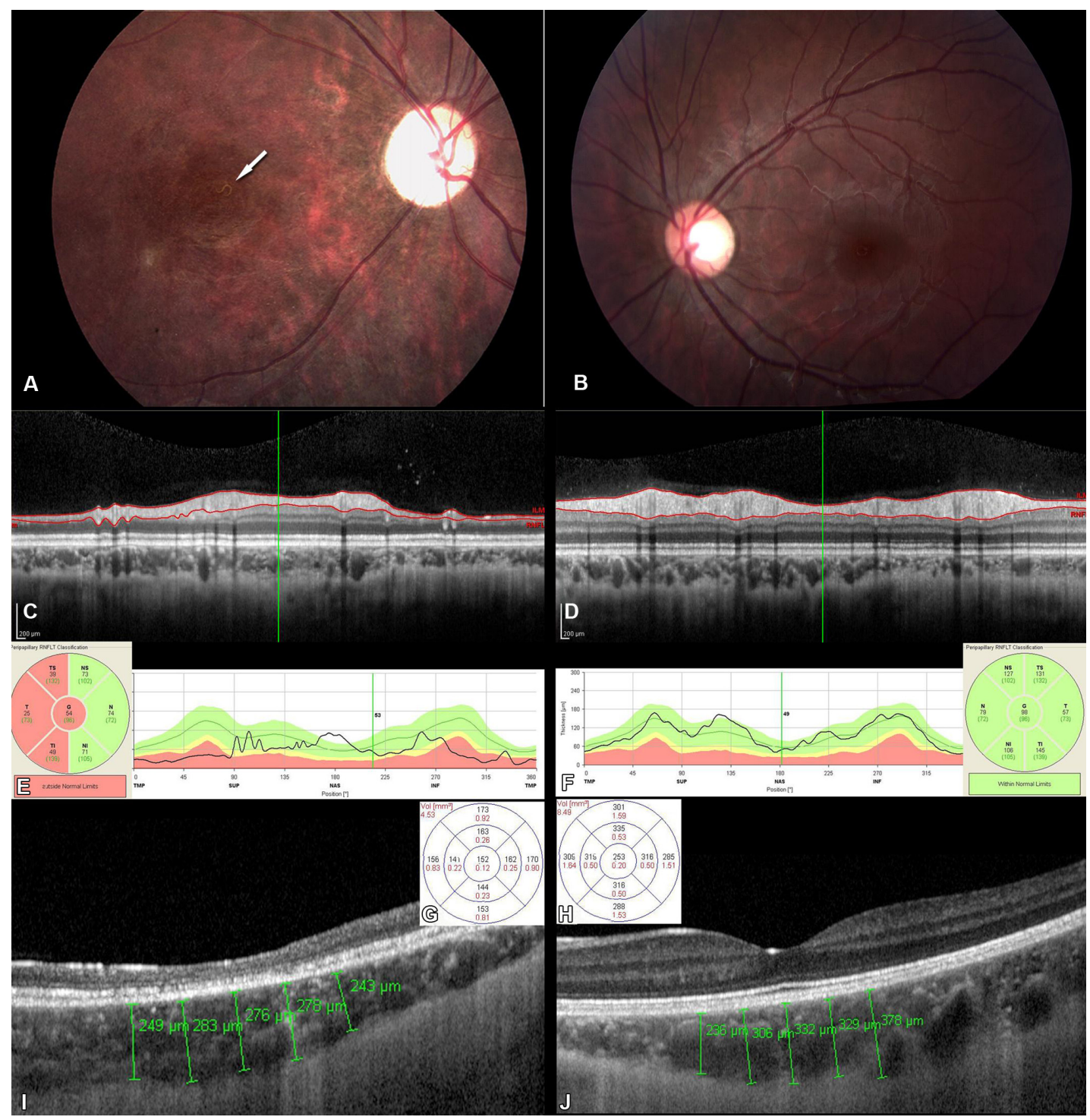

Figure 3 Chronic case (patient 3) with a worm (see white arrow) at the posterior pole (A, affected eye; B, contralateral eye) photocoagulated some years earlier. Note the atrophy of the retinal nerve fiber layer with a decrease in overall thickness of the layer (C, $\mathbf{E}$ and $\mathbf{D}$, F). Note also the substantial atrophy indicated by a decrease in mean macular thickness ( $\mathbf{G}$, affected eye; $\mathbf{H}$, contralateral eye), but without a significant change in thickness of the choroid (I, J), with some degree of outer retinal and photoreceptor disruption in the affected eye (I).

Abbreviations: TMP, temporal; SUP, superior; NAS, nasal; INF, inferior; RNFLT, retinal nerve fiber layer thickness; TS, temporal-superior; T, temporal; TI, temporalinferior; G, global average; NS, nasal-superior; N, nasal; NI, nasal-inferior.

This rare parasitic disease is relatively common in Brazil, preferentially affecting children and young adults. ${ }^{8}$ The differential diagnosis is hampered by the difficulty in finding the worm. ${ }^{9}$

OCT is a safe examination, without contraindications or risk of adverse effects, and is relatively easy to perform. OCT can help in cases where DUSN is suspected, aiding in the assessment of the retinal nerve fiber layer and areas of edema. $^{10}$

In one of the cases described (case 2), a motile worm was found at the posterior pole and evaluated by OCT. A hyper-reflective form was demonstrated, with localization in the outer retina but affecting all retinal layers (Figure 2).
Usually the worm is described as showing a subretinal localization. ${ }^{6,10,11}$ However, in contrast, evaluation of one case showed that the worm was not only present topographically in the outer retina, but was affecting all retinal layers. This corroborates the findings of another recent study, ${ }^{12}$ where the nematode was described as being in the inner retina on SD-OCT. Also, in the first series of cases of the disease published by Gass and Braunstein, two patients were described to have intraretinal localization of the nematode. ${ }^{1}$

In a previous study, our group demonstrated that some nematodes can be not only subretinal but also intraretinal; in one of the cases we described, time domain OCT (Stratus, 
Carl Zeiss Meditec, Dublin, CA) indicated that the worm was perpendicular to all layers of the retina. ${ }^{10}$

Gass suggested that the pathogenesis of DUSN appears to involve a local toxic effect on the outer retina caused by byproducts of the worm, and a more diffuse toxic reaction affecting both the inner and outer retinal tissues. In the latter, evanescent, gray-white outer retinal lesions result, and in the former, a rapid decrease in visual acuity and electroretinographic alterations occur, such as loss of ganglion cells with optic atrophy. ${ }^{13}$ We believe that the worm moves through all the retinal layers, not only the subretinal space. This may be the reason why we found few significant alterations in the choroid and a substantial retinal lesion in the retinal pigmented epithelium and retinal nerve fiber layer, with retinal layer thinning; all our cases showed considerable differences in macular and retinal nerve fiber layer thickness, possibly due to the toxic effect of the worm. The nematode causes chronic progressive changes in both retinal layers. For case 1, in whom finding the nematode was particularly difficult, it was possible to use another SD-OCT device (RTVue; Optovue Inc, Fremont, CA, USA), which identified a progressive loss in the ganglion cell layer after a few weeks. After destruction of the worm by laser, this loss was stabilized, but without anatomic recovery, even with improvement of visual acuity.

However, on examination of the choroid, we observed a smaller mean thickness in affected eyes, albeit not statistically significant, whereby some affected eyes had a higher value compared with the respective healthy eye.

Gass carried out a histopathologic study in a patient with DUSN and showed no evidence of an eosinophilic granulomatous reaction, but rather nongranulomatous vitritis, retinitis, and retinal and optic nerve perivasculitis, with extensive degeneration of the peripheral retina, mild degeneration of the posterior retina, mild optic atrophy, mild degenerative changes in the retinal pigmented epithelium, and a low-grade, patchy, nongranulomatous choroiditis, gliosis, loss of ganglion and receptor cell layers, and lymphocytic and plasma cell infiltration, most prominent in the inner retinal layer. ${ }^{13}$ In addition, the retinal receptor elements were intact throughout the posterior pole. This was also demonstrated in our patients by OCT, where slight difficulty was encountered in identifying the photoreceptor layer in some patients, but without marked defects seen, except in two patients treated in the chronic phase, who showed slight outer retinal and photoreceptor disruption (Figure 3, case 3). In contrast, a recent study described a lesion in the photoreceptors in the acute phase as a widespread outer retinal disruption which improved after treatment. ${ }^{12}$ Another study showed that the inner segment-outer segment junction was restored after treatment. ${ }^{14}$

The above findings, however, were not reproduced in our study. Even in the early phase, our patients did not display similar lesions in the photoreceptor layer. This was also observed by Garcia Filho et al who demonstrated that neither the early-stage nor late-stage disease patients showed focal or diffuse defects at the photoreceptor inner segment-outer segment junction. ${ }^{15}$

Histopathologically, no chorioretinal scars were found, and there was no evidence of choroidal atrophy; only a thinned retinal pigmented epithelium with irregularly depigmented and hyperpigmented areas was seen. ${ }^{16}$ In our patients, no marked alterations were found in the retinal pigmented epithelium, and it was not possible to identify changes in the choroid, with no statistically significant difference in mean thickness seen when compared with the healthy eye.

In a previous study, we showed the effect of this disease on the retinal nerve fiber layer using time-domain OCT and found significant atrophy, suggested that this could be a useful auxiliary test to distinguish DUSN from other mimicking diseases, such as toxoplasmosis in the punctate outer retinitis form, a disease that was previously studied by our group and in which no involvement of the retinal nerve fiber layer was found. ${ }^{10}$

Evaluation of patients in the acute phase showed retinal nerve fiber layer atrophy early in the initial stages which persisted even after treatment with photocoagulation, including in one patient who achieved a final visual acuity of 20/40 (Figure 1), despite considerable atrophy. In the two other cases ( 9 and 10), it was possible to perform earlier laser photocoagulation and to evaluate the outcome years after treatment. In spite of early treatment, these patients still had some degree of optic atrophy with an impact on visual acuity, albeit mild, compared with the contralateral eye. Retinal nerve fiber layer atrophy is an important feature of DUSN. ${ }^{10}$ Even a profound lesion was not accompanied by severe visual acuity loss, as in case 1, where the worm was discovered early and eradicated, resulting in good final visual acuity, but substantial decrease in retinal nerve fiber layer and macular thickness persisted.

Ophthalmic assessment using SD-OCT can help in evaluation of the changes caused by DUSN by contributing to an understanding of its pathogenesis, the differential diagnosis, and monitoring of the outcome of treatment. SD-OCT can be important for characterization of the clinical picture 
of DUSN, given that the worm cannot always be found, and therefore OCT features help to identify the disease. The most important OCT findings are neuroretinal atrophy and focal hyper-reflectivity through the retina in areas affected by the worm and a general loss of the inner retinal layer. In addition, progressive changes in the retinal nerve fiber layer can be monitored. Using this assessment technique, we believe that the nematode causes changes throughout the retina, mainly in the retinal nerve fiber layer and retinal pigmented epithelium, due to its toxic effects and its possible localization in all the layers of the retina not only the subretinal space, as demonstrated in other studies.

\section{Author contributions}

RFB, AMBC, ECDS, and MEF were involved in the design and conduct of the study, analysis and interpretation of data, and preparation, review, and approval of the manuscript. RFB and AMBC were involved in collection and management of the data. AMBC had full access to all the study data and takes responsibility for its integrity and for the accuracy of its analysis.

\section{Acknowledgment}

We thank Professor Tiemi Matsuo from the State University of Londrina for important comments, statistical analysis, and review, and Dr A Leyva, who helped with English editing of the manuscript.

\section{Disclosure}

No financial support was received for this submission. The authors have no conflicts of interest to report in relation to this work. This paper was presented in poster form at the 44th Annual Retina Society meeting held on September 21-25, 2011, Rome, Italy.

\section{References}

1. Gass JD, Braunstein RA. Further observations concerning the diffuse unilateral subacute neuroretinitis syndrome. Arch Ophthalmol. 1983; 101:1689-1697.

2. Souza EC, Casella AM, Nakashima Y, Monteiro ML. Clinical features and outcomes of patients with diffuse unilateral subacute neuroretinitis treated with oral albendazole. Am J Ophthalmol. 2005;140:437-445.

3. Casella AM, Farah ME, Belfort R Jr. Anthelminthic drugs in diffuse unilateral subacute neuroretinitis. Am J Ophthalmol. 1998;125:109-111.

4. Agarwal A. Diffuse unilateral subacute neuroretinitis. In: Agarwal A, editor. Gass' Atlas of Macular Diseases. 5th ed. Edinburgh, UK: Elsevier Saunders; 2012.

5. Souza EC, Casella AM. Clinical and tomographic features of macular punctate outer retinal toxoplasmosis. Arch Ophthalmol. 2009; 127:1390-1394.

6. Jesús MR, Isabel RC. Indocyanine green angiography for the detection of subretinal nematodes in diffuse unilateral subacute neuroretinitis (DUSN). Int Ophthalmol. 2004;25:295-297.

7. Spaide RF, Koizumi H, Pozonni MC. Enhanced depth imaging spectral-domain optical coherence tomography. Am J Ophthalmol. 2008; 146:496-500.

8. Souza, EC, Cunha S, Gass JD. Diffuse unilateral subacute neuroretinitis in South America. Arch Ophthalmol. 1992;110:1261-1263.

9. Souza EC, Abujamra S, Nakashima Y, Gass JD. Diffuse bilateral subacute neuroretinitis. Arch Ophthalmol. 1999;117:1349-1351.

10. Casella AM, Farah ME, Souza EC, Belfort R Jr, Oguido AP. Retinal nerve fiber layer atrophy as relevant feature for diffuse unilateral subacute neuroretinitis (DUSN): case series. Arq Bras Oftalmol. 2010;73: $182-185$.

11. Yusoff M, Alwi AA, Said MM, Zakariah S, Ghani ZA, Zunaina E. Intraocular nematode with diffuse unilateral subacute neuroretinitis: case report. BMC Ophthalmol. 2011;16:11-15.

12. Tarantola RM, Elkins KA, Kay CN, Folk JC. Photoreceptor recovery following laser photocoagulation and albendazole in diffuse unilateral subacute neuroretinitis. Arch Ophthalmol. 2011;129:669-671.

13. Gass JD, Scelfo R. Diffuse unilateral subacute neuroretinitis. J R Soc Med. 1978;71:95-111.

14. Goldberg N, Bhatnagar P. Letter to the editor. Diffuse unilateral subacute neuroretinitis (DUSN). Ocul Immunol Inflamm. 2012;20:139-142.

15. Garcia Filho CA, Soares AC, Penha FM, Garcia CA. Spectral domain optical coherence tomography in diffuse unilateral subacute neuroretinitis. J Ophthalmol. 2011;2011:285-296.

16. Gass JD, Gilbert WR Jr, Guerry RK, Scelfo R. Diffuse unilateral subacute neuroretinitis. Ophthalmology. 1978;85:521-545.
Clinical Ophthalmology

\section{Publish your work in this journal}

Clinical Ophthalmology is an international, peer-reviewed journal covering all subspecialties within ophthalmology. Key topics include: Optometry; Visual science; Pharmacology and drug therapy in eye diseases; Basic Sciences; Primary and Secondary eye care; Patient Safety and Quality of Care Improvements. This journal is indexed on

\section{Dovepress}

PubMed Central and CAS, and is the official journal of The Society of Clinical Ophthalmology (SCO). The manuscript management system is completely online and includes a very quick and fair peer-review system, which is all easy to use. Visit http://www.dovepress.com/ testimonials.php to read real quotes from published authors. 